\title{
IoT enable Low-Cost Implementation of Data Center Infrastructure Management System
}

\author{
Nguyen Trong Thuong ${ }^{1+}$, Yu Kun-Ming ${ }^{2}$ \\ ${ }^{1}$ Dept. of Computer Science and Informatics, Chung Hua University, nguyentrongthuong@live.com \\ ${ }^{2}$ Dept. of Computer Science and Informatics, Chung Hua University, yu@chu.edu.tw
}

\begin{abstract}
For any Data Center, environment and equipment monitoring for power and cooling in Data Center is very important. Thus, all Data Center need monitoring systems to help operators in their works. Currently, each type of equipment has their own monitoring system, provided by that equipment carrier. So, to monitoring whole devices in Data Center, people have to use many separated systems. To solve this issue, we apply IoT technique for connecting all devices into one real-time monitoring system, which help to save server resource and more convenience for operator.
\end{abstract}

Keywords: Data Center, Zigbee, Environment Monitoring, IoT, Modbus

\section{Introduction}

A data center (DC) is a building that host computing facilities like servers, routers, switches, firewalls. To keep these equipments in good working status, data center has to have supporting equipment such as: backup equipment, fire suppression facilities and cooling, power system [1, 2].

For any company, which have a data center, they have to build an engineer team to do the maintenance jobs. The main mission of those engineers is monitoring whole system include: server, storage, network devices and auxiliary equipment such as: Inrow cooling, PDU cabinet, UPS, STS system in real-time.

As we know, server, storage and network system have many software solutions for real-time monitoring and remotely control. But for supporting system, they do not have any general solution yet. Currently, maintainer could monitor some of those devices separately by connect to its portal through an IP address. However, this method has a lot of cons such as: no real-time function, could not retrieve log data in long period, cannot monitor many devices at the same time.

Another problem that engineers are facing is how to monitor environment condition in whole data center. Normally, they just check the humidity and temperature shown on cooling devices. In some cases, they have to go on-site and check those parameters by tools. Those actions or inefficiency or cost a lot of time.

Take into account of the case of Vietnam Technology \& Telecommunication JSC, they have a system call BMS to help DC administrators to monitoring temperature and humidity by show data of cooling devices in real-time, there are some power equipment are integrated in this system as well. This BMS help them a lot, however it has some problems: real-time data is not really real-time because data is collected cycle is 5 minutes. This problem caused by bottleneck issue of Modbus gateway. Besides that, humidity and temperature sensors are attached right at cooling devices output, so these parameters are always good. Thus, they have to check on-site every one hour to make sure environment condition is met requirement in entire DC, this action is obviously waste of time and labor power. If they do not have to check environment on-site, they will have more time for equipment maintenance to prevent error happen.

\footnotetext{
+ Corresponding author. Tel.: +8491190 4554;

E-mail address: nguyentrongthuong@live.com.
} 
Wireless sensor networks have common application in the domains like environmental sensing, health monitoring, home and building automation, smart energy, green computing, etc. WSN also able to build a good environment monitoring system for data center [3]. Thus, we applied IoT to build a Data Center Infrastructure Management system, which can help administrators to improve their working performance and help Data Center working more stable, less downtime.

Next section is related works, it includes summary of some other research papers on this field and short review about technologies that we are going use. Then it is followed by System Design section, where we describe about system architecture and its main components in here. Then, more detail about hardware setting up and method is shown in section IV System Implementation. Finally, we show results of this research by capture and explain about user interface of the DCIM system. Future work also presented in this part.

\section{Related Works}

As mentioned above, since Internet of Things become popular, especially Wireless Sensor Network, there are many researchers working on the field of applying WSN to monitor Data Center environment [3]. There are some papers published on IEEE Xplore website such as: Data Center Monitoring and Alerting System using WSN [3], Data Centre Temperature Monitoring with ESP8266 Based Wireless Sensor Network and Cloud Based Dashboard with Real Time Alert System [4], Smart Data Centre Monitoring System Based On Internet of Things (IoT) (Study Case: Pustipanda UIN Jakarta) [5].

Authors of the paper [3] applied Zigbee based sensors for implementing a monitoring system for data center, which can collect data of temperature and humidity in real-time and display on a website. Based on data gathered, they set up an alarm system that send SMS and email when parameters go beyond the threshold.

In 2017, another monitoring system with same feature had implemented, but they use a newer technology is ESP8622, which is enable data sending directly to cloud server by Wi-Fi connectivity. The advantage of this solution compare to the solution in previous paragraph is cheaper and simpler [4].

Paper [5] also proposes a solution by applying IoT to monitor the data center of the university. Their solution has a new function is monitoring Current beside of keep track environment parameters. In this project, they use DHT22 sensor to monitor environment, SCT 013 sensor to measure current of power line, these sensors are attached to an Arduino board. Arduino board read value from sensor and do some calculation before send data to the server through gateway, a Raspberry PI.

Three researches above conduct a good monitoring system for Data Center by applying IoT, however they are still lack of important function is integration of controlling and monitoring supporting equipment, as mentioned in Section 1. Therefore, we are motivated to do a research on applying IoT for a comprehensive monitoring system for Data Center, including: monitoring environmental factor, monitoring and controlling other sub-system in DC such as: Cooling, Power, Lighting.

In order to start the research, we have reviewed the most popular medium-range wireless communication for IoT system. Zigbee is an IEEE 802.15.4 based communication protocol, which is suitable to create personal area networks with small, low-power digital radios, such as for home automation, medical device data collection, and other low-power low-bandwidth needs, designed for small scale projects which need wireless connection [6]. It could be configured to work as one of those topology: star, mesh and cluster tree [7]

Another helpful technology that support for our research is Modbus. This is a serial communications protocol for use with its programmable logic controllers (PLCs). Modbus is often used to connect a supervisory computer with a remote terminal unit (RTU) in supervisory control and data acquisition (SCADA) systems [13].

\section{System Design}

After review previous researches and figure out the need of improvement, we worked with the data center operation team to clarify all type of equipment and its' communication protocol for proposing the 
system architecture as in Fig. 1. They also give a list of data corresponding to each devices that need to be showed on the website.

According to Fig. 1, this system could be divided into 4 blocks: Data Generation, Gateway, System Control and Client Monitoring. Data Generation include: Sensor; Cooling and Power equipment of Data Center; Industrial Fan Controlling and Sensing are covered in this part as well. For Gateway we use Latte Panda as the main gateway, where it receives data from Data Generation group via Zigbee communication then send received data to database server by Internet. SMS alarm is also included in this group. Next component of the system is System Control, where data is stored and web application is hosted. Here, entire data will go in/out Database Server through Web API service. Finally, the interface for users can interact with the system. It is a website with responsive feature enabled, so user can access by any device such as: Computer, Laptop, Smart Phone or tablet.

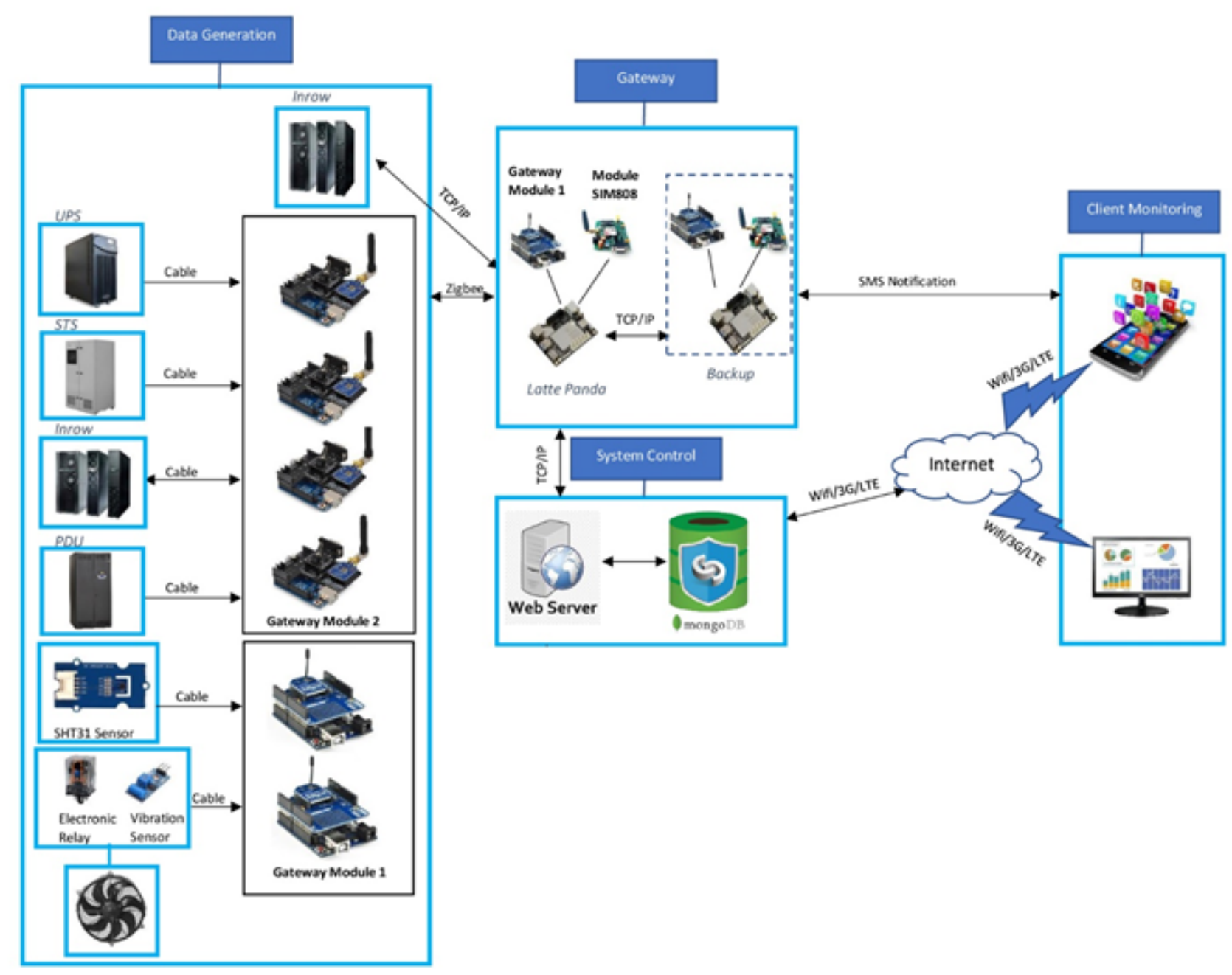

Fig. 1: System architecture.

\section{System Implementation}

According to designed architecture above, we deploy pilot system for testing and prove that our proposed technology can be used in a real Data Center. Following paragraphs describe about hardware module setting up and the area that we install IoT devices in Data Center.

As shown in Fig. 1, data is transferred from Data Generation modules to Gateway module via Zigbee network. The hardware includes a Module Xbee S2C attached to Arduino Uno R3 through an Arduino Xbee Shield. We use this combo to connect with below devices to be a send/receive module.

For connecting equipment in data center, such as inrow cooling, PDU cabinet, UPS, STS, to the gateway, we use Modbus RS485 module to connect devices to Arduino with Zigbee module attached, then the 
Arduino will send received data to the gateway. To read the data in these devices, we have to check Modbus Register Map of each device to know Register Number (Function Code [8]). This number will be used in the Arduino code, under library $<$ ModbusMaster.h $>$. By the way, we also need to know the SlaveID [8], which is set up in equipment, it is unique in the whole system.

As mentioned above, this data center has some industrial fans need to be monitored and remotely controlled. The idea for monitoring fans is checking if it is vibrating or not, because those fans are very strong so when it works, its' cover will shake. An alarm is sent if fan status is ON but vibration value is 0 . To control the fan, we use SignalR library to send data from server to relay in real-time without request from relay controller [9]. Temperature and humidity are measured by SHT31 sensor module. Because this sensor is high accuracy [10], which is important requirement for this system.

And the last module that we use in this project is SIM808, this module has two functions are GPS and GSM [11]. However, in this project, we only use this module to send SMS message as an alarm.

Main purpose of this project is help operation man to monitor whole system in real-time. We also plan to do data analysis later on. So, we choose Mongo Database because it highly supports real-time system and retrieve data very quick [12]. About security of database server, we set up a web API for connecting with the database, so application will not retrieve data directly from database server. Thus, we can block MongoDB port on the server. We also apply SSL certificate for web server to protect all of data.

As mentioned above, we build this system for eDatacenter of Vietnam Technology \& Telecommunication JSC. In this Data Center, there are many areas, but we choose Public Server Room area for testing the system. All Arduino modules is put on top of Racks and connect to Inrows and sensors. We attach sensors to front-door of racks because we commit with customers about temperature and humidity at this point.

\section{Results}

By deploying proposed technology, Data Center operation department can do their job on the website. Dashboard tab show them real-time data about Humidity and Temperature of connected devices and sensors In Fig. 2, we show real-time data of equipment as a map. If there are any error or warning, equipment icon in the map will change its color, operator can click these icons to see detail information. If people have need of reading log data for analysis or report, they can go to Power, Cooling and Environment tab then choose time range to see or export them to excel file.

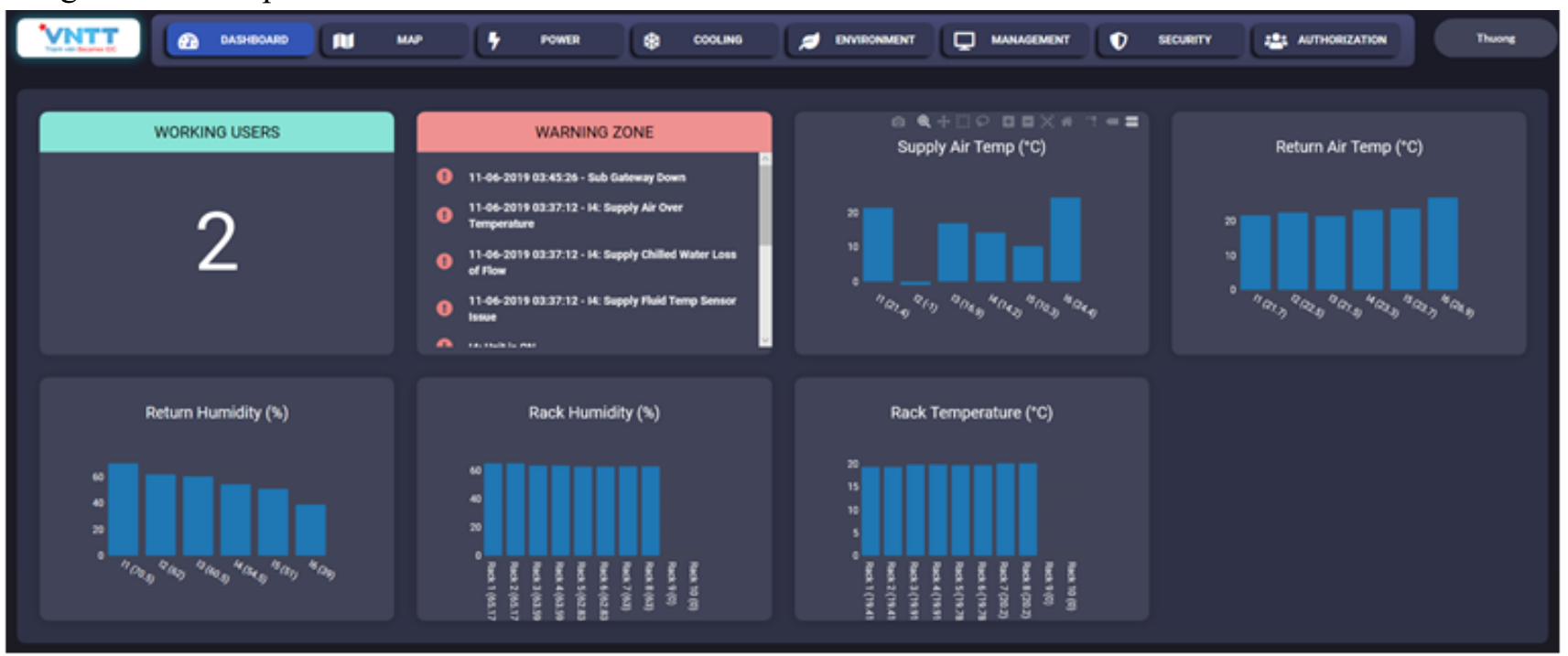

Fig. 2: Dashboard interface

Besides of giving basic functions to help operators, we also consider about user experience (UX). Thus, the web application is developed to work on mobile devices as well, then users do not need to stick with computer screen all the time, they can check information on their smart phone anytime, anywhere. In the other hand, we are developing an application for mobile device by using react native, which help us to create a cross-platform application for Android and iOS. The application has two main purpose: send error alarm 
and use for security purpose. For security purpose, the application helps us to take photo of customer, who access the Data Center, and also collect their signature to the database.

After three months of using new system, operation team gain a better work result. The total error in the first quarter of 2019 is half of this number in last year Table 1. The reason is this system have more sensing point and collect data more frequency about environment, so workers do not need to go on-site for checking environment too much. Thus, they have more time to check and do maintenance other devices, which are not monitored in this system.

Table 1: Experiment results

\begin{tabular}{|l|l|l|}
\hline & \multicolumn{1}{|c|}{ Old Monitoring System } & \multicolumn{1}{c|}{$\begin{array}{c}\text { New Monitoring } \\
\text { System }\end{array}$} \\
\hline Quantity of Sensor point & 6 sensors/10 rack & 2 sensors/10 rack \\
\hline Frequency of data collection & Every 5 minutes & Every 1 minutes \\
\hline $\begin{array}{l}\text { On-site checking environment } \\
\text { period }\end{array}$ & Every 1 hours (normal) & Every 2-3 hours \\
\hline Average error per quarter & Every 30 minutes (new system implement) & Every 1 hour \\
\hline
\end{tabular}

We also estimated total cost to implement this system for whole Data Center is around 4000 USD. It is much cheaper than other solution in the market. In the future, we will integrate Machine Learning in to this system for data analysis, which can help us optimize operation cost by reduce power consumption. And we also want to apply face detection for improving data center access security.

\section{Acknowledgements}

This paper is supported by Vietnam Technology \& Telecommunication JSC. They allow us to do testing on their Data Center and sponsor for equipment. We also want to thank website team, who help us to design and develop the website for showing data.

\section{References}

[1] J M. R. C. Truşcă , Ş. Albert and M. L. Soran , "The benefits of data center temperature monitoring," in 2015 Conference Grid, Cloud \& High Performance Computing in Science (ROLCG), Cluj-Napoca, Romania, 2016.

[2] "Techopdia," [Online]. Available: https://www.techopedia.com/definition/349/data-center. [Accessed 2702 2019].

[3] Kiran Nayak , Kaushik Nanda, Tulasi Dwarakanath , Hari Babu and David Selvakumar, "Data Centre Monitoring and Alerting System using WSN," in 2014 IEEE International Conference on Electronics, Computing and Communication Technologies (CONECCT), Bangalore, India, 2014.

[4] Saraswati Saha and Anupam Majumdar, "Data centre temperature monitoring with ESP8266 based Wireless Sensor Network and cloud based dashboard with real time alert system," in 2017 Devices for Integrated Circuit (DevIC), Kalyani, India, 2017.

[5] Feri Fahrianto, Nenny Anggraini , Hendra Bayu Suseno, Almas Shabrina and Alfatta Reza, "Smart data centre monitoring system based on Internet of Things (IoT) (study case: Pustipanda UIN Jakarta)," in 2017 5th International Conference on Cyber and IT Service Management (CITSM), Denpasar, Indonesia, 2017.

[6] "Wikipedia," [Online]. Available: https://en.wikipedia.org/wiki/Zigbee. [Accessed 0203 2019].

[7] Alexandru Lavric, Valentin Popa , Codrin Males and Ilie Finis , "A performance study of ZigBee wireless sensors network topologies for street lighting control systems," in 2012 International Conference on Selected Topics in Mobile and Wireless Networking, Avignon, France, 2012.

[8] Sadik Tamboli , Mallikarjun Rawale, Rupesh Thoraiet and Sudhir Agashe, "Implementation of Modbus RTU and Modbus TCP communication using Siemens S7-1200 PLC for batch process," in 2015 International Conference on Smart Technologies and Management for Computing, Communication, Controls, Energy and Materials (ICSTM), Chennai, India, 2015.

[9] Liu Ye , "The Development of Production Safety Gridding Supervision System," in 2014 Fifth International 
Conference on Intelligent Systems Design and Engineering Applications, Hunan, China, 2014.

[10] "Digital Humidity Sensor SHT3x (RH/T)," Sensirion, [Online]. Available: https://www.sensirion.com/en/environmental-sensors/humidity-sensors/digital-humidity-sensors-for-variousapplications/. [Accessed 0203 2019].

[11] U Bharavi and Rao M Sukesh, "Design and development of GSM and GPS tracking module," in 2017 2nd IEEE International Conference on Recent Trends in Electronics, Information \& Communication Technology (RTEICT), Bangalore, India, 2017.

[12] Mayur M Patil , Akkamahadevi Hanni , C H Tejeshwar and Priyadarshini Patil , "A qualitative analysis of the performance of MongoDB vs MySQL database based on insertion and retriewal operations using a web/android application to explore load balancing - Sharding in MongoDB and its advantages," in 2017 International Conference on I-SMAC (IoT in Social, Mobile, Analytics and Cloud) (I-SMAC), Palladam, India, 2017.

[13] "Wikipedia," [Online]. Available: https://en.wikipedia.org/wiki/Modbus. [Accessed 0203 2019]. 